\title{
reviscafuences
}

ISSN: 1575.7072 | 8.15SN: 2172.7775

Páginas: $77-90$

Recibido: 2020-06-11

Revisado: 2020-12-18

Aceptado: 2021-01-11

Preprint: 2021-01-15

wWw.revistascientificas.us.es/index.php/fuentes/index

Publicación Final: 2021-01-31

DOI: https://doi.org/10.12795/revistafuentes.2021.v23.i1.12024

\section{Cuando el aprendizaje basado en la práctica profesional no provoca un interés laboral}

\author{
When learning based on professional practice does not lead to an interest in work
}

\author{
(iD Ricard Calvo Palomares \\ Universitat de València (España) \\ (iD) Raúl Payá Castiblanque \\ Universitat de València (España) \\ (iD) Enric Sigalat Signes \\ Universitat de València (España)
}

\begin{abstract}
La vinculación entre lo académico y lo profesional se ha convertido en un elemento esencial para impulsar la calidad educativa de la Universidad actual. El presente artículo muestra una experiencia de innovación docente en educación superior basada en el uso de tecnologías profesionales llevadas al ámbito educativo. Concretamente del aplicativo informático y la metodología utilizados profesionalmente por CEAL-Asociación Española de Auditores Sociolaborales en el desarrollo de su labor, y que ha permitido profesionalizar la docencia y aproximarla a la realidad del colectivo profesional. Con objeto de conocer la percepción del alumnado acerca de la propuesta metodológica y su potencial transferencia al ámbito profesional se empleó un cuestionario. Además, se incorporó la visión de las empresas participantes preguntándoles sobre su posible transferencia. Se hallaron resultados ambivalentes. Por un lado, el alumnado valoró la propuesta metodológica como altamente positiva, útil, aplicada y práctica, pero, por otro lado, no percibieron que la actividad como auditor/a sociolaboral sea una posible salida profesional futura, cuando es una de las 7 salidas profesionales establecidas en el Libro Blanco para el Grado de Relaciones Laborales y Recursos Humanos. En conclusión, el efecto positivo en la esfera educativa no tuvo el impacto deseado en la esfera profesional debido a factores externos relacionados con el contexto empresarial, ya que, ninguna de las empresas colaboradoras había realizado previamente ninguna auditoría sociolaboral, ni conocían las herramientas y métodos para efectuarla.
\end{abstract}

\section{Abstract}

The link between the academic and the professional has become an essential element in promoting the educational quality of today's University. This article shows an experience of teaching innovation in higher education based on the use of professional technologies brought to the educational field. Specifically, the computer application and methodology used professionally by CEAL-Spanish Association of Socio-Labour Auditors in the development of their work, and which has allowed the professionalization of teaching and bringing it closer to the reality of the professional collective. A questionnaire was used to find out the students' perception of the methodological proposal and its potential transfer to the professional field. In addition, the vision of the participating companies was incorporated by asking them about their possible transference. Ambivalent results were found. On the one hand, the students valued the methodological proposal as highly positive, useful, applied and practical, but, on the other hand, they did not perceive that the activity as a sociolabour auditor is a possible future professional outlet, when it is one of the 7 professional outlets established in the White Paper for the Degree in Labour Relations and Human Resources. In conclusion, the positive effect in the educational sphere did not have the desired impact in the professional sphere due to external factors related to the business context, since none of the collaborating companies had previously carried out any socio-labour audit, nor were they aware of the tools and methods for carrying it out. 
Palabras clave

software profesional, profesionalización, aprendizaje, práctica profesional, universitarios, auditoría sociolaboral, metodología.

Keywords

professional software, professionalization, learning, professional practice, universities, socio-labour audit, methodology.

\section{Introducción: el aprendizaje basado en vincular lo académico con lo profesional y/o viceversa}

Si bien toda innovación educativa responde a la necesidad de implementar un cambio estructural e implica modificar aspectos esenciales del sistema educativo utilizado hasta el momento, no podemos olvidar que se trata de aspectos que actúan y se implementan desde lo micro, desde lo concreto de una docencia en un aula concreta (Aguerrondo, 2002). Tres son los elementos clave a tener en cuenta por un docente en su proceso de puesta en práctica de experiencia de innovación en el aula: el objetivo perseguido, las particularidades del contexto de aplicación y la evaluación de los resultados de la propuesta (Torío-López et al., 2010).

En cuanto al objetivo perseguido, la propuesta que presentamos trata de vincular la escuela con la vida, la teoría con la práctica, lo académico con lo profesional o las aulas con la realidad, planteándose como aspectos necesarios en la docencia actual (Bellucci y Pennacchio, 2016; Hewitt-Dundas, Gkypalib y Roperb, 2019). Una pedagogía que apuesta por la combinación adecuada de formación en competencias tecnológicas (técnicas) con la formación en competencias laborales como elemento clave y diferenciador del éxito de las acciones emprendidas en el aula (Abreu, 1997; Abreu y Soler, 2015; López-Martínez, 2016). Por lo que establecer una metodología que permita poner en práctica lo aprendido es clave, saber usar los conocimientos en la práctica, así como aplicar lo aprendido. El aprendizaje basado en vincular lo académico con lo profesional ( $\mathrm{y} / \mathrm{o}$ viceversa), también nombrado en algunos momentos como aprendizaje profesionalizado (Ortiz-Ocaña, 2003), aprendizaje profesionalizador, aprendizaje profesional o incluso aprendizaje con orientación profesional (Secadas, 1962), no difiere de los planteamientos tradicionales de buscar un cambio y transformación que ocurre en quien aprende. Lo que lo diferencia serán los mecanismos y las metodologías implementadas para ello, las condiciones pedagógicas en las que se produce, el rol que asume quien aprende y quien enseña, los resultados finales de ese cambio (transformación), y las peculiaridades que adquiere dicho proceso de aprendizaje, y más teniendo en cuenta el contexto de una educación que no puede desligarse de la sociedad digital y de los continuos cambios (Trostinskaia et al., 2017). Dejar los parámetros tradicionales, para enseñar a aprender la aplicación de conocimientos teóricos a casos aplicados, con supuestos de trabajo propuestos por el profesorado extraídos de la realidad o buscados incluso directamente por el alumnado (Ayoujil-Serrano, 2017). Proponiendo la generación de marcos de referencia, restando importancia a la mera adquisición de procesos de trabajo aplicados al pie de la letra (Mahecha, 2012). Aprender a aprender, enfocar los contenidos al uso y la práctica, conectar el aprendizaje con unas metas profesionales. Todo conocimiento es reflejo del mundo circundante. Se debe enseñar a los estudiantes a fundamentar teóricamente lo desarrollado en la práctica. Reflexionar desde y para la acción. Enseñar a los alumnos a fundamentar teóricamente lo desarrollado en la práctica, vinculando lo teórico con lo práctico, pero sin olvidar la vinculación de lo práctico con lo teórico. Pues siguiendo a Pedró (2020), cualquier profesión se puede beneficiar mucho de una formación orientada al aprendizaje profesionalizador, y que sea dual, o sea, que, pueda tener lugar ésta en el lugar de trabajo. Así mismo, los universitarios también pueden tener una mayor perspectiva y un mayor conocimiento de las exigencias del mercado de trabajo (Sánchez y Leicea, 2007) y una mayor (mejor) retroalimentación (Ahea, 2016).

Aprendizaje que intenta transmitir que el profesionalismo (ser profesional) es una actitud ante el trabajo y los alumnos necesitan de ella (Grañeras y Parras, 2008). En este sentido, el docente es un instructor, un docente profesional, que propone al alumno la oportunidad de pasar por una instancia previa a la puesta en práctica de las competencias adquiridas. Que propone un contacto constante con la realidad, con las áreas profesionales y las comunidades profesionales de referencia. El profesorado asume un rol de líder profesional en el proceso educativo (Eirín, García y Montero, 2009). Se trata de un aprendizaje importante para el alumnado participante, formativo, con contenidos que permitan su crecimiento profesional, con un alto grado de transferencia y aplicabilidad, proactivo y potenciador de competencias, tanto técnicas como laborales (Ortiz-Ocaña, 2011) y de experiencias de práctica técnica y profesional (Grey, 1997). En este sentido, la inclusión del aprendizaje profesionalizador conlleva el desarrollo de una "evaluación auténtica" en la medida que la experiencia de innovación docente trata de llevar a cabo "tareas auténticas" en la esfera educativa, con atributos o características similares o iguales a las que se encontrará el alumnado en el desarrollo de su trabajo en la esfera profesional (Barrientos-Hernán et al., 2020). 


\subsection{La experiencia de innovación desarrollada}

Presentamos una experiencia docente novedosa implementada desde hace más de 5 cursos académicos, que ha intentado aproximar las dimensiones académica y profesional en una asignatura como Técnicas de Auditoría Sociolaboral [de $3^{\circ}$ curso del Grado de Relaciones Laborales y Recursos Humanos (GRLRH)] a través de la propuesta de una serie de prácticas y actividades en las que el alumnado tenía que utilizar como elementos básicos para el desarrollo de su trabajo, el uso de documentos y metodología propuesta por CEALAsociación Española de Auditores Sociolaborales, asociación profesional representativa del sector y que la utilizan en el desarrollo profesional de las mismas. Que además viene siendo evaluada desde hace 3 cursos académicos, a través de una encuesta de valoración y satisfacción del alumnado participante. Se trata de una experiencia desarrollada en el contexto de aplicación de las Ciencias Laborales, contexto en el que no ha sido sencillo ni habitual la implementación de experiencias de innovación docente. Los estudios efectuados por INVESLAB-UV (2010, 2014 y 2016) ponen de manifiesto las principales dificultades o resistencias con las que han convivido estos estudios en su evolución (Calvo et al., 2017):

- Ausencia/inexistencia de un departamento propio, específico, que velara por fomentar la titulación, y a sus egresados.

- Derivado de ello, la ausencia de profesorado oriundo -profesional- de las Ciencias Laborales en la titulación y en general en la universidad, ha influido en que las propuestas de innovación docente carecieran en la mayoría de los casos de una empatía profesional que aumentara su aplicabilidad.

- Y la orientación extremadamente aplicada de la profesión. Que ha impedido que se reflexionara y se teorizara desde este ámbito, y con esta perspectiva. Ello responde a la escasa trayectoria investigadora de carácter específico habida hasta el momento desde y para las Ciencias Laborales.

Por todo ello, en el contexto de las Ciencias Laborales, resulta clave apostar por una metodología innovadora que propugne aproximar lo teórico -académico- a lo aplicado- profesional, y/o viceversa. Principalmente la iniciativa, se ha concretado en la propuesta y uso de las herramientas técnicas utilizadas en el ejercicio profesional como auditores/as sociolaborales como herramientas de uso docente. Y ello, tanto para el desarrollo de prácticas puntuales realizadas en el aula sobre algún aspecto en concreto de la auditoría, como de soporte para la realización del trabajo práctico final planteado, consistente en el desarrollo de una auditoría sociolaboral a una determinada realidad -organización, empresa o cualquier entidad que pudiera ser auditada sociolaboralmente- (tabla 1).

\section{Tabla 1}

Resumen de la propuesta docente realizada

\begin{tabular}{|c|c|c|}
\hline Objetivo & Fases & Aspecto profesional utilizado ${ }^{a}$ \\
\hline $\begin{array}{l}\text { Práctica final. } \\
\text { Aplicación práctica } \\
\text { del proceso de } \\
\text { auditoría a un } \\
\text { supuesto real }\end{array}$ & $\begin{array}{l}\text { Fase de pre-auditoría (contactos iniciales) } \\
\text { Fase de diseño (objetivos, alcance y } \\
\text { metodología) Fase de ejecución (recogida de } \\
\text { las informaciones) Fase de análisis Fase de } \\
\text { cierre (elaboración del informe final) }\end{array}$ & $\begin{array}{l}\text { Contrato o carta de encargo Presupuesto } \\
\text { Cronograma Flujograma Plan de auditoría } \\
\text { Check-list (áreas de riesgo) Papeles de } \\
\text { trabajo Informe final (párrafos de opinión) } \\
\text { Carta de manifestaciones }\end{array}$ \\
\hline
\end{tabular}

Para ello, nos propusimos la realización de una práctica final que aplicara todo aquello desarrollado a lo largo del cuatrimestre en clase. Así, planteamos la realización de una auditoría sociolaboral, utilizando la metodología y documentación que los profesionales de las auditorías sociolaborales utilizan en sus despachos (firmamos un convenio de colaboración con CEAL para el uso académico de sus materiales, documentos y metodología). Para que fuera lo más aplicada posible, propusimos que cada grupo de trabajo -equipo auditorbuscara un caso en el que desarrollar la misma. Es decir, un supuesto de aplicación -organización, pública o privada, grande, mediana o pequeña, con o sin ánimo de lucro- real sobre el que desarrollar la intervención de auditoría de manera completa, y conforme a la tabla 1. Propuesta práctica que mucho tiene que ver con las competencias establecidas en la guía docente de la asignatura, ya que básicamente con ella se cumplía con la mayoría de los resultados del aprendizaje establecidos en la misma, entre los que destacamos los siguientes: conocer los conceptos, características y tipología de la auditoría sociolaboral, conocer el marco normativo y regulador aplicable a la auditoría sociolaboral y su tipología, identificar y analizar las causas que justifiquen una auditoría, entre otros. Por lo que respecta al tercero de los elementos establecidos como clave, la evaluación de la experiencia docente. Ésta experiencia se implantó en el curso 2014-2015 y desde hace tres cursos, se empezó a medir sus efectos a través de la evaluación de la percepción-satisfacción del alumnado con la misma. 


\subsection{Objetivos}

Partiendo de la experiencia de innovación educativa implementada en los tres últimos cursos en el GRLRH, en la presente investigación nos proponemos valorar la incidencia del uso docente de la metodología profesional y su aplicativo informático CEAL-Pro en una triple dimensión: a) comparar la satisfacción de los alumnos entre los distintos cursos académicos para identificar sí las mejoras incluidas en el último curso académico han tenido incidencia y han permitido mejorar la práctica realizada; b) conocer el grado de satisfacción y las dificultades percibidas por los alumnos en la realización del ejercicio práctico en las aulas con objeto de medir el grado de incidencia de la experiencia innovadora tanto en su dimensión macro (valoración global de la asignatura en la cual se ha realizado la práctica), meso (utilidad práctica para el ejercicio profesional del aplicativo informático) y micro (utilidad del informe científico-técnico resultante de la auditoria sociolaboral); y, c) finalmente, tras identificar el éxito de aprendizaje en la esfera educativa, nos proponemos indagar la percepción de los alumnos y las empresas sobre la transferencia del aprendizaje en la esfera profesional.

\section{Metodología}

\subsection{Participantes}

Se recogen las opiniones de seis grupos de estudiantes de los últimos tres cursos académicos de la asignatura con una población de 175 alumnos (tabla 2). El trabajo de campo fue realizado en las últimas dos clases de cada curso académico, que consistía, por un lado, en la explicación por parte del profesorado del cuestionario de evaluación de la práctica de auditoría sociolaboral realizada a través del aplicativo informático profesional CEAL-Pro y, por otro lado, la realización de la evaluación del alumnado a través de la plataforma virtual de la universidad para garantizar el anonimato de los estudiantes y evitar que contestaran aquello socialmente aceptado (deseabilidad). Así mismo, en el último curso también se solicitó a los alumnos que preguntaran a las empresas en las que habían desarrollado sus trabajos finales de auditoría sobre su percepción.

Tabla 2

Muestra del estudio

\begin{tabular}{|c|c|c|c|c|c|}
\hline Curso & Grupo & Matriculados $\left(\mathbf{N}^{\circ}\right)^{a}$ & $\begin{array}{c}\text { Media } \\
\text { asistencia } \\
\text { clase }\end{array}$ & $\begin{array}{c}\text { Respuestas } \\
\text { obtenidas ( } \mathbf{N}^{\circ} \text { ) }\end{array}$ & $\begin{array}{l}\% \text { respuesta } \\
\text { obtenida }^{c}\end{array}$ \\
\hline \multirow{2}{*}{ 2017-2018 } & A & 46 & 39.2 & 35 & 89.3 \\
\hline & B & 43 & 39.6 & 32 & 80.8 \\
\hline \multirow{2}{*}{ 2018-2019 } & $A$ & 52 & 38.4 & 27 & 70.3 \\
\hline & C & 57 & 45.1 & 37 & 82.0 \\
\hline \multirow{2}{*}{ 2019-2020 } & $A$ & 43 & 32,3 & 24 & 74.3 \\
\hline & B & 47 & 35,4 & 20 & 56.6 \\
\hline Totales & & 288 & 230 & 175 & 76.1 \\
\hline
\end{tabular}

a Utilizando documentos y materiales procedentes de la metodología desarrollada por CEAL-Asociación Española de Auditores Sociolaborales, siendo el producto resultante el aplicativo informático profesional CEAL-Pro.

${ }^{b}$ Número de alumnos que han contestado el cuestionario de evaluación.

${ }^{c}$ Calculado respecto de la media de asistencia.

\section{Número de alumnos matriculados}

\subsection{El cuestionario como instrumento de evaluación}

Para evaluar la incidencia del uso del aplicativo informático profesional en la docencia universitaria se utilizó un cuestionario. En los dos primeros cursos académicos (2017-2018 y 2018-2019) el cuestionario semiestructurado contenía siete preguntas destinadas a conocer el grado de utilidad de la práctica realizada y el grado de satisfacción con los métodos, prácticas, contenidos y materiales de soporte utilizados (tabla 3). En este sentido, desde un punto de vista teórico, la satisfacción debe de ser entendida como las necesidades que experimentan las personas sobre un bien, servicio o producto determinado y la respuesta adecuada del proveedor sobre dichas necesidades, intereses o expectativas (Pérez-Juste, 2000). En el ámbito educativo, 
el alumnado es el destinatario del servicio, y su satisfacción vendría a relacionarse con el proceso educativo y el grado en el que la institución (la Universidad) atiende a sus expectativas, intereses y necesidades (GentoPalacios y Vivas-García, 2003). Existe diferentes dimensiones teóricas relacionadas con la satisfacción del alumno universitario, tales como, el sistema de enseñanza o de trabajo, la organización académica, la vida universitaria y las infraestructuras y servicios universitarios o la seguridad vital, económica y emocional del alumnado (Álvarez-Botello y Reyes-Pérez, 2015; Mejías y Martínez, 2009; Jiménez-González et al., 2011). Para el desarrollo del cuestionario se seleccionó únicamente las dimensiones correspondientes al sistema de enseñanza o de trabajo. Con objeto de simplificar el número de preguntas a realizar se seleccionaron las subdimensiones de metodología de enseñanza, el contenido de los programas, los materiales de soporte disponibles en el aula y las practicas efectuadas. Para dar validez al contenido del constructo de satisfacción, se utilizó el juicio de los 6 profesores expertos que impartieron la asignatura de auditoria sociolaboral en cada grupo de estudiantes. Tras su recomendación, se incluyeron a las cinco preguntas cerradas, dos abiertas para conocer mejor las percepciones del alumnado. Finalmente, la fiabilidad del instrumento de medida fue medido a través del coeficiente Alfa de Cronbach, ya que se trata de uno de los indicadores más utilizadas para comprobar la consistencia interna de las variables de una misma dimensión (Prat y Doval, 2003). El resultado obtenido fue de $\alpha=0.964$, por lo que la homogeneidad de las cuatro preguntas correspondientes a la dimensión de satisfacción fue muy elevada.

Tabla 3

Cuestionario de evaluación cursos 2017-2018 y 2018-2019

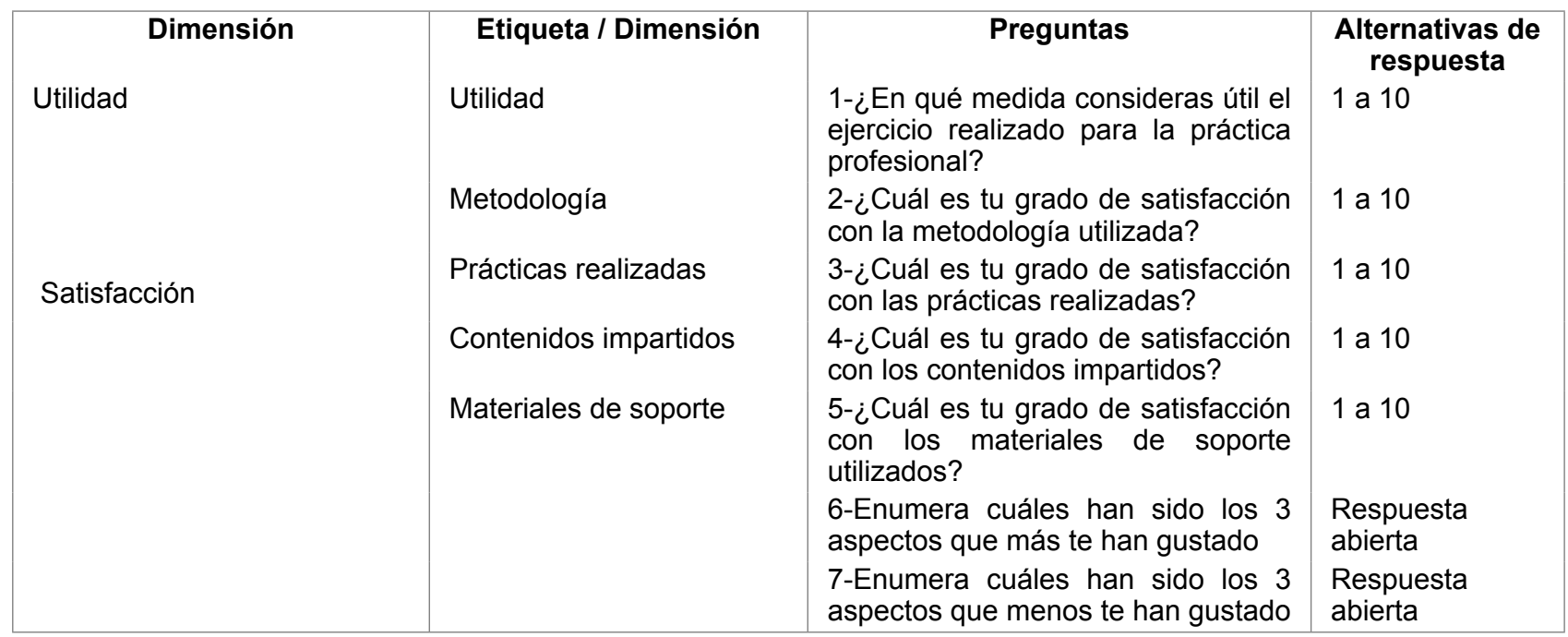

Los resultados obtenidos en los dos primeros registros (curso 2017-2018 y 2018-2019), mostraron resultados moderados en las cinco dimensiones cuantitativas analizadas, con una media de 6,43 sobre 10 en la utilidad práctica del aplicativo informático y un grado de satisfacción moderado $(6,32$ en la metodología utilizada; 6,66 en las prácticas realizadas; 6,20 en los contenidos impartidos; y, 6,09 en los materiales de soporte) (Calvo, Payá y Sigalat, 2019). Así mismo, los elementos cualitativos recogidos de las preguntas abiertas mostraron dos dimensiones que podrían explicar el impacto moderado y las dificultades encontradas. La primera, referente a las dificultades de los alumnos en comprender el lenguaje técnico del aplicativo profesional CEAL-pro y, la segunda, el excesivo trabajo que conlleva el aprendizaje de la herramienta profesional y su aplicación en el ámbito empresarial. Con objeto de mejorar la práctica en las aulas, el profesorado ha elaborado nuevos materiales de soporte para facilitar el aprendizaje del alumnado en el uso del aplicativo informático como también facilitar la comprensión del lenguaje técnico utilizando a través de la elaboración de un diccionario. Es por ello, que el primero de los objetivos de la presente investigación es conocer si las mejoras introducidas han surgido efecto y han mejorado el grado de satisfacción de los alumnos.

Como se puede observar, en el último curso académico (2019-2020) se ha incorporado al cuestionario ocho nuevas preguntas para medir la incidencia de la práctica educativa tanto en la esfera académica como en la esfera profesional (tabla 4). Las construcciones de las dimensiones fueron desarrolladas en base a los constructos teóricos utilizados por diversos autores para medir la capacitación para la inserción al mundo laboral y especialización profesional del alumnado (González-López, 2003; Mejías y Martínez, 2009; Saraiva, 2008) y validadas por los mismos 6 profesores expertos consultados en la primera edición del cuestionario. De este modo, relacionado con el segundo de los objetivos de la presente investigación vinculado con el grado de 
incidencia en la esfera educativa, se ha preguntado al alumnado sobre tres ámbitos: a) un ámbito macro, b) un ámbito meso y c) un ámbito micro. El Alfa de Cronbach resultante para dicha dimensión fue de $\alpha=0.964$. Para finalizar, en referencia al tercer objetivo correspondiente a la incidencia en la esfera profesional, se recogió tanto la visión del alumnado como también la visión de las empresas participantes, observando de este modo la capacidad de transferencia del conocimiento adquirido en las aulas al ejercicio profesional.

Tabla 4

Preguntas incorporadas en el curso académico 2019-2020

\begin{tabular}{|c|c|c|c|}
\hline Dimensión & EtiquetaDimensión & Preguntas & Alternativas de respuesta \\
\hline \multirow[t]{3}{*}{$\begin{array}{l}\text { Incidencia en la esfera } \\
\text { educativa }\end{array}$} & Macro & $\begin{array}{l}\text { ¿Qué valoración global } \\
\text { haces sobre lo aprendido } \\
\text { en la asignatura? }\end{array}$ & 1 a 10 \\
\hline & Meso & $\begin{array}{l}\text { ¿Consideras que la } \\
\text { práctica realizada ha } \\
\text { mejorado tu aprendizaje? }\end{array}$ & 1 a 10 \\
\hline & Micro & $\begin{array}{l}\text { ¿Consideras que el informe } \\
\text { científico-técnico resultante } \\
\text { de la práctica ha mejorado } \\
\text { tu aprendizaje? }\end{array}$ & 1 a 10 \\
\hline \multirow[t]{2}{*}{$\begin{array}{l}\text { Incidencia en la esfera } \\
\text { profesional (visión del } \\
\text { alumnado) }\end{array}$} & Salida profesional & $\begin{array}{l}\text { ¿En qué medida } \\
\text { consideras que la auditoria } \\
\text { sociolaboral puede ser } \\
\text { buena salida profesional? }\end{array}$ & 1 a 10 \\
\hline & Dificultades & $\begin{array}{l}\text { ¿Cuál crees que puede } \\
\text { ser la principal dificultad } \\
\text { a las que se enfrentan } \\
\text { los profesionales de la } \\
\text { auditoria sociolaboral? }\end{array}$ & $\begin{array}{l}\text { A-El desconocimiento } \\
\text { empresarial. } \quad \text { B-Falta } \\
\text { de una ley que obligue } \\
\text { realizarla. C- Falta de } \\
\text { procesos estandarizados. } \\
\text { D-Sin dificultades }\end{array}$ \\
\hline \multirow[t]{3}{*}{$\begin{array}{l}\text { Incidencia en la esfera } \\
\text { profesional (visión } \\
\text { empresarial) }\end{array}$} & Auditoria previa & $\begin{array}{l}\text { ¿Ha realizado con } \\
\text { anterioridad una auditoria } \\
\text { sociolaboral? }\end{array}$ & Sí / No \\
\hline & Conocimiento & $\begin{array}{l}\text { ¿Conoces las herramientas } \\
\text { de auditoria sociolaboral? }\end{array}$ & Sí / No \\
\hline & Contratación & $\begin{array}{l}\text { ¿Después de conocer el } \\
\text { trabajo de los alumnos te } \\
\text { plantearías la posibilidad } \\
\text { de contratarlos en el futuro } \\
\text { para realizar una autoría } \\
\text { sociolaboral de carácter } \\
\text { profesional? }\end{array}$ & Sí / No \\
\hline
\end{tabular}

\subsection{Análisis estadísticos}

Partiendo de los objetivos planteados se realizaron diferentes análisis estadísticos. En referencia al primer objetivo, se efectuó la prueba T de Student para muestras independientes que se utiliza para comparar las medias de dos grupos de casos, es decir, a los efectos de nuestra investigación, dicho análisis nos permitirá comparar los resultados del primer curso académico (2017-2018) con el segundo (2017-2019) y, el primero con el tercero (2019-2020). De esta manera, conoceremos si las medidas incluidas en el último curso académico han mejorado la práctica y uso del aplicativo informático CEAL-Pro.

Para estudiar el segundo de los objetivos, se realizaron diversas operaciones estadísticas. En primer lugar, se realizó un análisis descriptivo a través del cálculo del coeficiente $\mathrm{R}$ de Pearson, con un nivel de confianza del $95 \%$ ( $p$ valor $=<.005$ ) para conocer las asociaciones entre los elementos de incidencia en la esfera académica (macro, meso y micro) y la satisfacción del alumnado en las distintas dimensiones de la práctica profesional realizada (metodología, prácticas realizadas y materiales de soporte utilizados). Finalmente, aquellas dimensiones que resultaron estadísticamente significativas, fueron incluidas en un modelo de regresión lineal múltiple para determinar la influencia relativa de cada dimensión de participación 
sobre las variables dependientes correspondientes a la incidencia de la práctica profesional tanto a nivel macro, meso y micro. Para finalizar con la esfera educativa, se efectuó una triangulación metodológica en la medida que se analizaron los resultados cualitativos de las preguntas abiertas ( 3 ventajas y 3 desventajas) y se compararon con los análisis estadísticos realizados.

En referencia al último de los objetivos relacionados con la medición de la incidencia de la apuesta educativa en la esfera profesional se realizaron tres análisis: a) se efectuaron los mismos análisis descriptivos y de regresión lineal efectuados para estudiar el segundo de los objetivos, pero, en esta ocasión, se estudió la relación entre la pregunta etiquetada como salida profesional (variable dependiente) (tabla 5) con las cuatro dimensiones de satisfacción; b) además, se realizó un análisis descriptivo con objeto de conocer la visión de los alumnos en cuanto a las dificultades (etiqueta dificultades; tabla 5) encontradas para trasferir los conocimientos adquiridos en las aulas a la esfera profesional; y c) finalmente, se efectuó un análisis descriptivo de la visión empresarial recogidas en las tres preguntas efectuadas (auditoria previa, conocimiento y contratación; ver tabla 5). Para efectuar los análisis estadísticos se utilizó el programa estadístico SPSS -versión 26.

\section{Resultados}

\subsection{Mejora de la práctica profesional efectuada}

En la siguiente tabla 5, se pueden observar los resultados obtenidos tras comparar las medias entre el primer curso que se implementó la práctica profesional y los siguientes cursos académicos. De los resultados obtenidos se puede identificar cómo la percepción de utilidad del alumnado del primer curso $(M=6.40 ; D T=1.71)$ no fue mejorada en el segundo curso $(M=6.47 ; \mathrm{DT}=1.62 ; \mathrm{T}=.226, \mathrm{p}=.822)$. Sin embargo, tras incluir las nuevas instrucciones, la percepción de utilidad mejoró considerablemente en los alumnos de la última promoción (2019-2020) $(M=7.16 ; D T=1.22 ; T=2,54, p=.012)$. Situación similar podríamos encontrar en los elementos de satisfacción, ya que, salvo en la dimensión correspondiente a la práctica realizada con una puntuación moderada en el primer curso $(M=6.66$; $D T=1.83)$ con ligero descenso no significativo en el segundo $(M=6.61$; $\mathrm{DT}=1.77 ; \mathrm{T}=-.150, \mathrm{p}=.881)$ e incremento no estadísticamente significativo en el tercero $(M=6.91 ; D T=1.16$; $\mathrm{T}=.813, \mathrm{P}=.418$ ). Las otras tres dimensiones de satisfacción se vieron incrementadas en la promoción 20192020, tras introducir las mejoras en el ejercicio práctico profesional. En este sentido, se observa en la tabla 5 cómo mientras que el grado de satisfacción con la metodología empleada no varió entre el primer curso y el segundo, sí se vio fuertemente incrementada en el último curso, como también, el grado de satisfacción con las dimensiones correspondiente a los contenidos impartidos y los materiales de soporte empleados.

\section{Tabla 5}

Comparación de medias de los tres cursos académicos

\begin{tabular}{|c|c|c|c|c|c|c|c|}
\hline \multirow[t]{2}{*}{ Dimensión } & \multirow{2}{*}{$\begin{array}{c}\text { Curso } \\
\text { 2017-2018 } \\
\text { M (DT) }{ }^{a}\end{array}$} & \multirow{2}{*}{$\begin{array}{c}\text { Curso } \\
\text { 2018-2019 } \\
\text { M (DT) }{ }^{a}\end{array}$} & \multirow{2}{*}{$\begin{array}{c}\text { Curso } \\
\text { 2019-2020 } \\
\text { M (DT) }\end{array}$} & \multicolumn{2}{|c|}{$\begin{array}{l}\text { Comparación medias } \\
(2017-2018)-(2018-2019)\end{array}$} & \multicolumn{2}{|c|}{$\begin{array}{l}\text { Comparación medias } \\
(2017-2018)-(2019-2020)\end{array}$} \\
\hline & & & & $\mathbf{T}^{b}$ & $\mathbf{P}$-valor ${ }^{c}$ & $\mathbf{T}^{b}$ & $\mathbf{P}$-valor ${ }^{c}$ \\
\hline Utilidad & $6.40(1.71)$ & $6.47(1.62)$ & $7.16(1.22)$ & .226 & .822 & 2.543 & .012 \\
\hline Metodología & $6.21(1.68)$ & $6.45(1.68)$ & $6.93(1.11)$ & .831 & .408 & 2.511 & .013 \\
\hline $\begin{array}{l}\text { Prácticas } \\
\text { realizadas }\end{array}$ & $6.66(1.83)$ & $6.61(1.77)$ & $6.91(1.16)$ & -.150 & .881 & .813 & .418 \\
\hline $\begin{array}{l}\text { Contenidos } \\
\text { impartidos }\end{array}$ & $6.16(1.60)$ & $6.25(1.51)$ & $6.80(1.13)$ & .350 & .753 & 2.268 & .025 \\
\hline $\begin{array}{l}\text { Materiales } \\
\text { de soporte }\end{array}$ & $5.98(1.49)$ & $6.22(1,50)$ & $6.80(1.02)$ & .895 & .373 & 3.146 & .002 \\
\hline
\end{tabular}

${ }^{a} \mathrm{M}=$ media de la promoción; DT= desviación típica.

${ }^{b} \mathrm{~T}=$ prueba $\mathrm{T}$ de Student.

${ }^{c}$ P-valor= significancia al $95 \%$.

\subsection{La medida de la incidencia en la esfera académica}

En referencia al segundo de los objetivos, los resultados descriptivos correspondientes al último curso académico, han mostrado un impacto positivo del uso de la metodología profesional y el aplicativo informático en la esfera académica tanto en la valoración global de la asignatura (visión macro) con una puntuación media de 6.89 sobre $10(\mathrm{DT}=1.12)$, como en la utilidad de la práctica del uso de la herramienta profesional CEAL 
pro (visión meso) con un media de $7.16(\mathrm{DT}=1.22)$ y la utilidad del informe científico resultante del proceso de auditoria proporcionado por el aplicativo informático (visión micro) $(M=6.77 ; D T=1.05)$. En referencia a las relaciones de las dimensiones de satisfacción e incidencia, en el análisis de correlación realizado (tabla 6), se ha identificado como la valoración positiva de la asignatura está muy relacionada con el grado de satisfacción de los alumnos tanto en los métodos empleados para utilizar el aplicativo informático $(r=.908 ; p=.000)$ como en la propia práctica $(r=.902 ; p=.000)$, los contenidos $(r=.930 ; p=.000)$ y materiales de soporte utilizados $(r=$ $.827 ; p=.000$ ). Además, también se han hallado relaciones similares entre el éxito a nivel meso y micro con las cuatro dimensiones de satisfacción.

Tabla 6

Análisis de correlación entre las medidas de incidencia en la esfera educativa y satisfacción del alumnado

\begin{tabular}{|c|c|c|c|c|c|c|c|}
\hline & Macro & Meso & Micro & Metodología & $\begin{array}{l}\text { Prácticas } \\
\text { realizadas }\end{array}$ & $\begin{array}{l}\text { Contenidos } \\
\text { impartidos }\end{array}$ & $\begin{array}{c}\text { Materiales } \\
\text { de } \\
\text { soporte }\end{array}$ \\
\hline Macro & 1 & & & & & & \\
\hline Meso &, $709^{* *}$ & 1 & & & & & \\
\hline Micro &, $861^{\star *}$ &, $681^{* *}$ & 1 & & & & \\
\hline Metodología &, $908^{* *}$ &, $919^{* *}$ &, $903^{* *}$ & 1 & & & \\
\hline Prácticas realizadas &, $902^{* *}$ &, $875^{\star *}$ &, $822^{* *}$ &, $900^{* *}$ & 1 & & \\
\hline Contenidos impartidos &, $930^{* *}$ &, $920^{* *}$ &, $896^{* *}$ &, $894^{\star *}$ &, $836^{* *}$ & 1 & \\
\hline Materiales de soporte &, $827^{* *}$ &, $872^{* *}$ &, $861^{* *}$ & $858^{\star *}$ &, $787^{\star *}$ &, $923^{* *}$ & 1 \\
\hline
\end{tabular}

**. La correlación es significativa en el nivel 0,01 (bilateral).

Los modelos resultantes del análisis de regresión múltiple (tabla 7), han podido constatar que la satisfacción de los alumnos en el uso del aplicativo informático vendría a explicar el $91.4 \%$ de la incidencia en la valoración global de la asignatura (macro) ( $\mathrm{r}^{2}$ ajustado $\left.=.914 ; \mathrm{F}=115.115, \mathrm{p}=.000\right)$, el $60,7 \%$ en la utilidad de las prácticas realizadas a través de la metodología CEAL pro (meso) ( $r 2$ ajustado $=.607 ; F=17.588, p=.000$ ) y el $85,9 \%$ de la utilidad del informe científico-técnico resultante de su uso (micro) $r^{2}$ ajustado= .859; $F=66.381, p=.000$ ). No obstante, ateniéndose al valor de $\beta$ y su significancia, se puede observar que la satisfacción de los alumnos con la metodología empleada y los contenidos impartidos son las dos dimensiones más relevantes para explicar el grado de incidencia en la esfera educativa. En este sentido, se observa que por cada unidad más puntuada sobre la satisfacción en la metodología empleada se incrementará 3.39 décimas la valoración global de la asignatura, 8.94 décimas la utilidad percibida de la práctica y 4.11 décimas la percepción de utilidad del informe científico-técnico. Mientras que para los niveles de satisfacción en los contenidos impartidos se incrementará en 4.14 décimas la valoración global de la asignatura y 3.58 la percepción de utilidad de la práctica efectuada por cada punto más valorado sobre la satisfacción en los contenidos.

Tabla 7

Análisis de regresión múltiple para medir los determinantes de incidencia en la esfera educativa

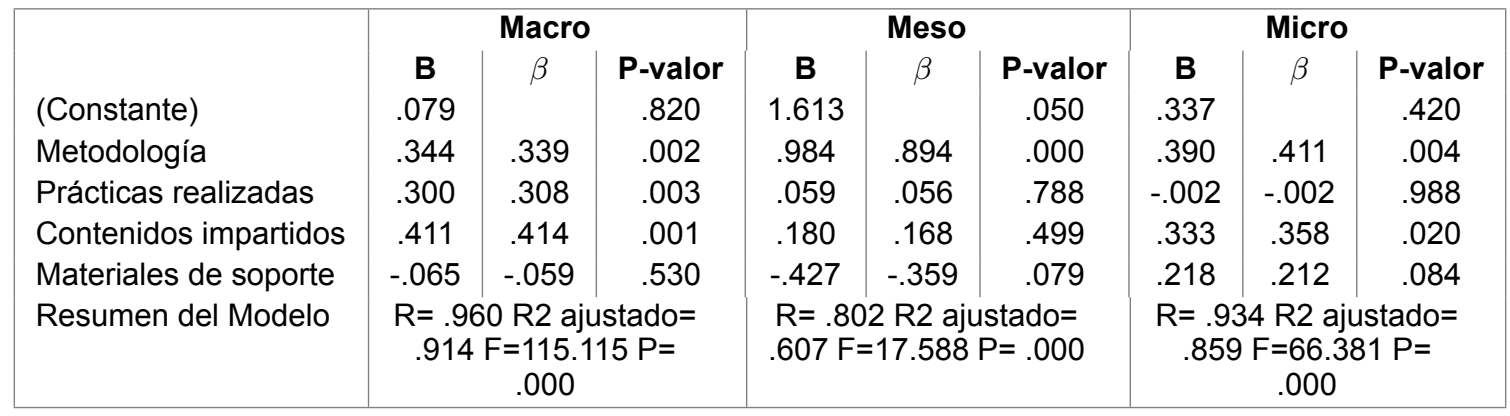

Los resultados cuantitativos obtenidos vendrían a confirmarse en las citas textuales recogidas en las preguntas abiertas planteadas y que recogemos en la tabla 8 . En este sentido, se observa un grado de satisfacción elevado de los alumnos con los métodos y contenidos empleados en la utilización del aplicativo informático de uso profesional valorando que es práctico, real, innovador y que se transfiere a un mejor aprendizaje. No obstante, a pesar de haber mejorado la práctica realizada elaborando guías y diccionarios, tal y como se ha podido observar en la comparación de medidas de los distintos cursos académicos (tabla 5), lo cierto es que el alumnado sigue considerando que los ejercicios realizados presentan una elevada carga de trabajo y 
consideran que tienen un nivel de abstracción considerable. Además, consideran que es difícil la transferencia de lo aprendido en las aulas al tejido empresarial, siendo una cuestión ligada con el último de los objetivos de la presente investigación que se desarrolla en el siguiente apartado.

Tabla 8

Análisis de las citas textuales de los alumnos del curso 2019-2020

\begin{tabular}{|c|c|c|c|}
\hline \multicolumn{2}{|l|}{ Aspectos positivos } & \multicolumn{2}{|l|}{ Aspectos a mejorar } \\
\hline Dimensión & Citas textuales & Dimensión & Citas textuales \\
\hline Enfoque práctico y real & $\begin{array}{l}\text { "muy práctica", "muy } \\
\text { aplicado", "bastante real", } \\
\text { "muchos ejemplos reales", } \\
\text { "vinculado con la profesión", } \\
\text { "prácticas muy reales", } \\
\text { "interesante visión de } \\
\text { metodología aplicada", }\end{array}$ & Carga de trabajo & $\begin{array}{l}\text { "muy exigente", "mucho } \\
\text { trabajo" "demasiadas } \\
\text { prácticas", "prácticas, } \\
\text { trabajo, dedicación", } \\
\text { "necesitas mucho esfuerzo" } \\
\text { "demasiado esfuerzo para } \\
\text { aprobarla" }\end{array}$ \\
\hline Innovación & $\begin{array}{l}\text { "sus contenidos no los } \\
\text { conocía", "es algo nuevo", } \\
\text { "es novedoso y útil", "es una } \\
\text { posibilidad de futuro", "son } \\
\text { cosas nuevas" }\end{array}$ & Nivel de abstracción & $\begin{array}{l}\text { "es complicado", "me } \\
\text { ha costado, nos ha } \\
\text { dado demasiada } \\
\text { libertad", "desconocido y } \\
\text { complicado", "no te podias } \\
\text { despistar" "la novedad } \\
\text { hace que dudes" }\end{array}$ \\
\hline $\begin{array}{l}\text { Visión positiva de las } \\
\text { clases y profesor }\end{array}$ & $\begin{array}{l}\text { "genera aprendizaje, me } \\
\text { ha gustado", "sesiones } \\
\text { amenas, sabe del tema } \\
\text { (todos los textos eran } \\
\text { suyos)", "las clases han } \\
\text { sido muy dinámicas", "hemos } \\
\text { aprendido" }\end{array}$ & Dificultad de trasferencia & $\begin{array}{l}\text { "difícil que las empresas } \\
\text { hagan auditorías", "lo veo } \\
\text { difícil de hacer en las } \\
\text { empresas" "buscar una } \\
\text { empresa que quiera es } \\
\text { complicado" "difícil que las } \\
\text { empresas quieran hacer } \\
\text { una auditoria" }\end{array}$ \\
\hline
\end{tabular}

\subsection{La medida de la incidencia en la esfera profesional}

A diferencia de las preguntas que miden la incidencia en la esfera educativa con puntuaciones en torno a 7 sobre 10, los resultados registrados para observar en qué medida perciben los alumnos que pueden transferir los conocimientos y habilidades adquiridos en las aulas con el uso del aplicativo informático a la esfera profesional se observó un descenso en la puntuación media, siendo de 6.48 sobre 10 (DT=0.95). Los resultados moderados obtenidos podrían ser explicados por la percepción de los alumnos sobre las dificultades de transferencia que como se observa en la figura 1 ningún estudiante considero que no existían dificultades, mientras que el $61,47 \%$ consideraron que la mayor dificultad vendría dada por el desconocimiento empresarial, lo que vendría confirmar las dificultades descritas en las preguntas abiertas (tabla 9). Además, el 23,26\% del estudiantado consideró que la falta de una ley que determine la obligación a efectuar una auditoria sociolaboral también supone una importante barrera para la transferencia.

Por lo que respecta a las dificultades percibidas por los egresados y egresadas en Ciencias Laborales trabajen como auditores/as sociolaborales, el análisis de correlaciones (tabla 9) entre los niveles de satisfacción con la práctica de innovación docente realizada a través de la metodología profesional CEAL y que los estudiantes se planteen trabajar en el futuro como auditores laborales ha mostrado una relación positiva en las cuatro dimensiones de satisfacción. Ello vendría a confirmar el potencial de transferencia de la práctica efectuada. En concreto, al considerar la auditoría como posible futuro profesional ha resultado relacionado tanto con la satisfacción en la metodología empleada $(r=.803 ; p=.000)$ y la propia práctica realizada $(r=.652 ; p=.000)$ como con los contenidos $(r=.718 ; p=.000)$ y los materiales de soporte utilizados $(r=.722 ; p=.000)$. 


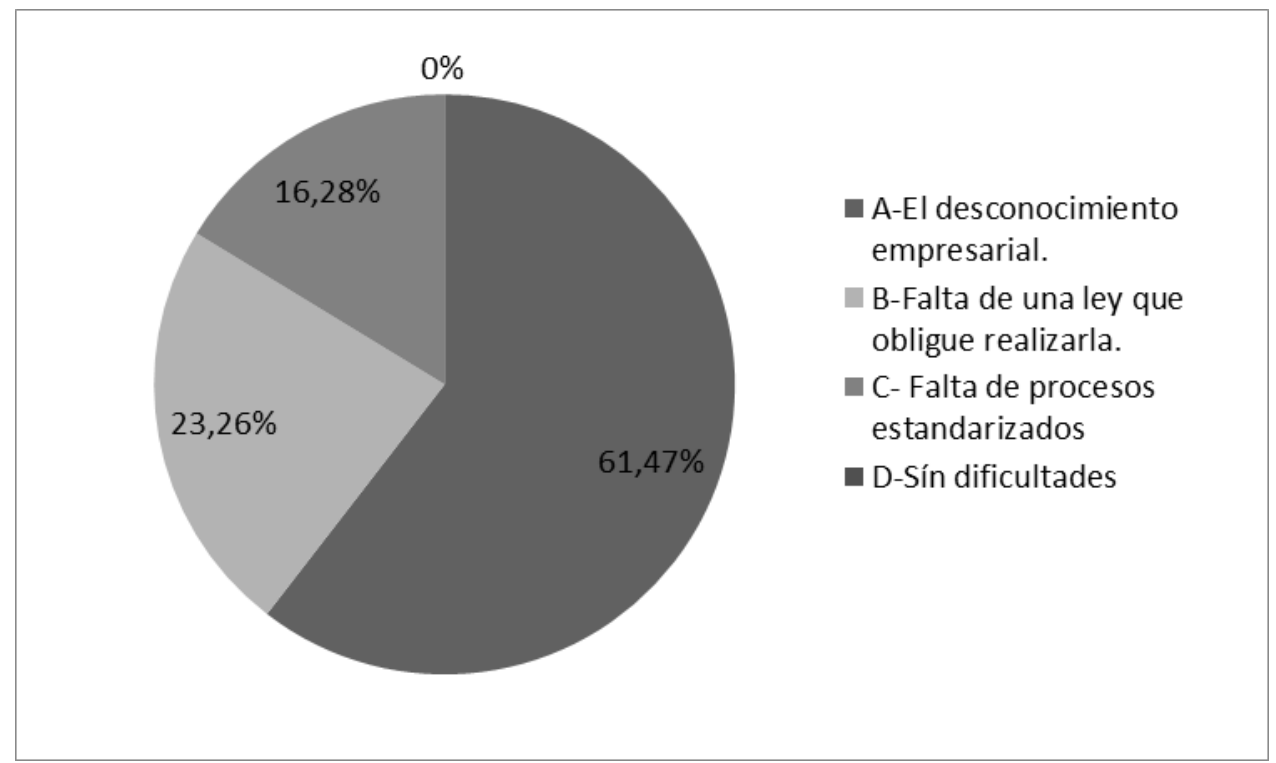

Figura 1. Dificultades de transferencia de la esfera educativa a la profesional

Tabla 9

Análisis de correlación entre las medidas de incidencia en la esfera profesional y satisfacción del alumnado

\begin{tabular}{|l|c|c|c|c|c|}
\hline & Salida profesional & Metodología & $\begin{array}{c}\text { Prácticas } \\
\text { realizadas }\end{array}$ & $\begin{array}{c}\text { Contenidos } \\
\text { impartidos }\end{array}$ & $\begin{array}{c}\text { Materiales } \\
\text { de soporte }\end{array}$ \\
Salida profesional & 1 & 1 & & \\
Metodología &, $803^{* *}$ &, $900^{* *}$ & 1 & \\
Prácticas realizadas &, $652^{* *}$ &, $894^{* *}$ &, $836^{* *}$ & 1 & \\
Contenidos impartidos &, $718^{* *}$ &, $858^{* *}$ &, $787^{* *}$ &, $923^{* *}$ & 1 \\
Materiales de soporte &, $722^{* *}$ & & & \\
\hline
\end{tabular}

**. La correlación es significativa en el nivel 0,01 (bilateral). 
No obstante, en el análisis de regresión múltiple se observó (tabla 10), de nuevo, al igual que sucedió en la esfera educativa, cómo el elemento clave fue la propia metodología empleada basada en el uso de herramientas profesionales en el entorno universitario. En este sentido, se observó que por cada unidad más puntuada sobre la satisfacción en la metodología empleada se incrementará en 7.11 décimas la percepción del alumnado sobre la posibilidad de trabajar en el futuro como auditores/as sociolaborales. De hecho, el modelo resultante vendría a determinar que los niveles de satisfacción explicarían el $65,5 \%$ de dicho planteamiento de futuro profesional $\left(r^{2}\right.$ ajustado $\left.=.655 ; F=18.498, p=.000\right)$.

Tabla 10

Análisis de regresión múltiple para medir los determinantes de la incidencia en la esfera profesional

\begin{tabular}{l|c|c|c|} 
& Salida profesional & & \\
(Constante) & B & $\beta$ & P-valor \\
Metodología & 1,542 & &, 016 \\
Prácticas realizadas &, 610 &, 710 &, 003 \\
Contenidos impartidos &,- 107 &,- 130 &, 525 \\
Materiales de soporte &, 052 &, 062 &, 798 \\
Resumen del Modelo &, $161 \quad \mathrm{R}=.809 \mathrm{R} 2$ ajustado= &, $655 \mathrm{~F}=18.489 \mathrm{P}=.000$ &, 383 \\
\hline
\end{tabular}

\section{Discusión y conclusiones}

La presente investigación tuvo por objeto valorar la incidencia del uso de herramientas profesionales en la esfera educativa. La primera de las cuestiones tratadas reveló que, tras las dos primeras ediciones del cuestionario las mejoras implementadas en el uso del aplicativo informativo han resultado satisfactorias. En segundo lugar, en cuanto al grado de incidencia en la esfera académica y profesional, se han obtenido resultados ciertamente paradójicos, dado que el alumnado valida la propuesta innovadora, valorando positivamente su utilidad y su aplicabilidad, que vendría a coincidir con los hallazgos de estudios previos (Álvarez-Botello y Reyes-Pérez, 2014; Jiménez González, Terriquez-Carrillo y Robles-Zepeda, 2011; Mejías y Martínez, 2009). Pero, sin embargo, no perciben ni abogan por la actividad como auditores/as sociolaborales como una posible salida profesional futura pese a que es una de las 7 salidas profesionales establecidas en el Libro Blanco del Título de Grado en Ciencias Laborales y Recursos Humanos de la ANECA (ANECA, 2005). Estudios previos han identificado una importante transferencia del conocimiento generado en la esfera académica hacia el tejido productivo empresarial (Bellucci y Pennacchio, 2016; Hewitt-Dundas, Gkypalib y Roperb, 2019), pero, sin embargo, nuestros hallazgos han obtenido resultados contradictorios. ¿Por qué las buenas valoraciones de la propuesta docente no se transforman en un mayor interés profesional por la profesión vinculada con la auditoría sociolaboral? A nuestro entender, el alumnado es un reflejo de lo que ocurre a su alrededor. En este sentido se confirman los factores determinados en un estudio realizado con anterioridad por Calvo y Rodríguez (2014), en el que se establecían tres bloques de factores que dificultaban la utilización de la auditoría: a) factores internos del proceso de auditoría; b) factores propios de las organizaciones en las que van a ser aplicadas; $y, c$ ) factores del contexto social.

Así pues, los problemas de transferencia de la esfera educativa a la profesional, vendrían dados por factores estructurales del tejido empresarial y mercado laboral, más que por factores internos propios de la experiencia de innovación educativa. De hecho, el resultado de las tres preguntas (ver tabla 5) efectuadas a las 12 empresas donde los alumnos han desarrollado el ejercicio práctico en el curso 2019-2020, así lo han puesto de manifiesto, en la medida que, ninguna empresa había realizado previamente ninguna auditoría sociolaboral ni conocían las herramientas ni los métodos para efectuarla. Además, tras realizar la práctica solo 2 de las 12 empresas, se plantearía la posibilidad de contratar a los alumnos en el futuro para realizar una autoría sociolaboral de carácter profesional, lo que vendría a confirmar las dificultades descritas por el propio alumnado.

Ello obliga a continuar trabajando en esta línea y en intensificar una labor de promoción conjunta con la asociación profesional (CEAL) con la finalidad de dar a conocer las potencialidades de la auditoría sociolaboral. Campaña de sensibilización tanto con el alumnado -futuros usuarios e implementadores- como con las Asesorías y Despachos profesionales y sus empresas clientas - potenciales usuarios-.

Junto a ello, no podemos obviar la necesidad de que las Administraciones públicas muestren un interés especial por la materia. Buenos ejemplos recientes de ello, son la obligación desde mayo de 2019 de que las empresas de más de 250 trabajadores sean auditadas salarialmente con la finalidad de luchar contra la brecha salarial, 


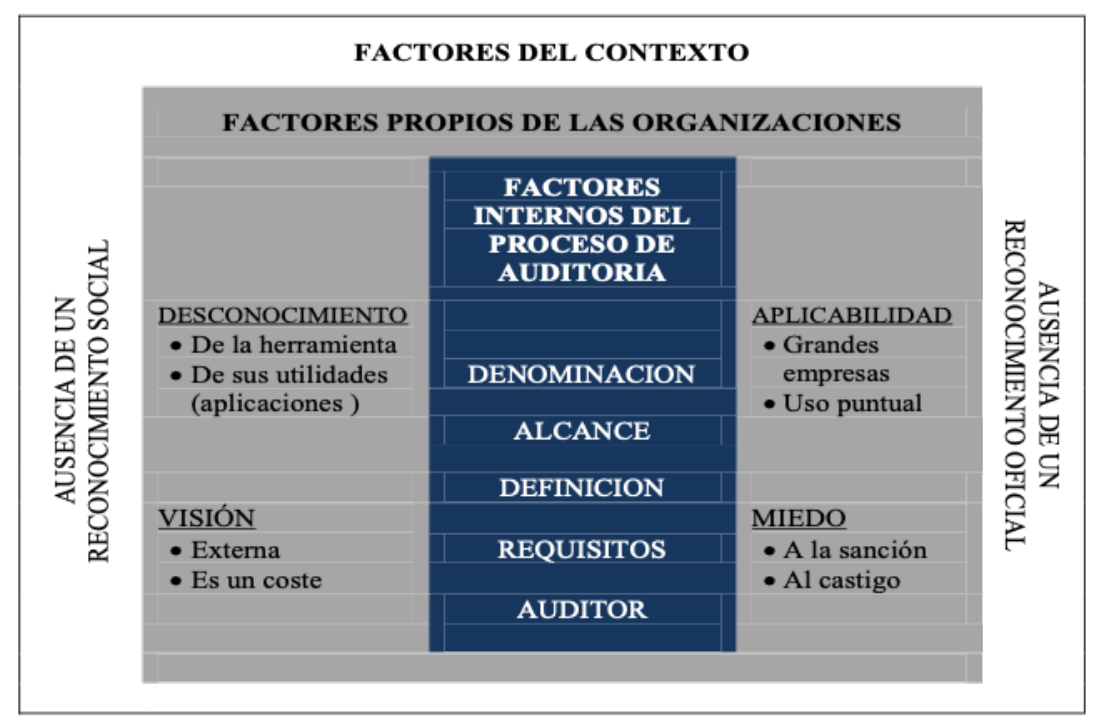

Figura 2. Factores que dificultan la utilización de la auditoría sociolaboral

o la decisión del Gobierno Vasco recientemente de crear junto a CEAL, el Instituto de Auditoría Laboral e Igualdad que tiene por objeto mejorar la cultura del cumplimiento normativo por parte de las empresas y la consecución de los objetivos de la Agenda 2030 en el ámbito de las relaciones laborales y en especial al ODS 8 que hace referencia al Trabajo Decente.

\section{Transferencia}

Dada la temática de la experiencia de innovación docente planteada (vincular lo profesional con la docencia) consideramos que el proceso de transferencia articulado adquiere una gran importancia en el currículo docente del alumnado, que adquiere competencias formativas de carácter aplicado y con proyección en el mercado de trabajo.

\section{Apoyos}

Proyecto de Innovación Docente "Innovació Universitària en Ciències Laborals. El primer pas cap a la millora profesional" (proyecto UV-SFPIE_PID-19-1095903), aprobado por el Vicerrectorado de Políticas de Formación y Calidad Educativa de la Universitat de València para el curso 2019-2020 y a los Convenios de Colaboración firmados entre CEAL-Asociación Española de Auditores Sociolaborales y la Universitat de València (a través de INVESLAB-UV).

\section{Referencias}

Abreu, R. (1997). Pedagogía profesional: Una propuesta abierta a la reflexión y al debate. La Habana.

Abreu, R., y Soler, J. (2015). Didáctica de las Especialidades de la Educación Técnica y Profesional. La Habana: Pueblo y Educación.

Aguerrondo, I. (2002). La escuela del futuro I. Cómo piensan las escuelas que innovan. Buenos Aires: Papers Editores.

Ahea, M. (2016). The Value and Effectiveness of Feedback in Improving Students' Learning and Professionalizing Teaching in Higher Education. Journal of Education and Practice, 7(16), 38-41.

Álvarez Botello, J., Chaparro-Salinas, E. M., y Reyes-Pérez, D. E. (2015). Estudio de la Satisfacción de los Estudiantes con los Servicios Educativos Brindados por Instituciones de Educación Superior del Valle de Toluca. REICE. Revista Iberoamericana Sobre Calidad. Eficacia Y Cambio En Educación, 13(2), 5-26. 
ANECA. (2005). Libro Blanco del Título de Grado en Ciencias Laborales y Recursos Humanos. Madrid: Aneca. Ayoujil-Serrano, O. (2017). Hacia la mejora de la competencia emprendedora en educación secundaria: un estudio piloto en la ciudad de Melilla. Publicaciones de la Facultad de Educación y Humanidades del Campus de Melilla, 47, 59-77.

Barrientos-Hernán, E. J., López-Pastor, V. M., y Pérez-Brunicardi, D. (2020). Evaluación Auténtica y Evaluación Orientada al Aprendizaje en Educación Superior. Una Revisión en Bases de Datos Internacionales. Revista Iberoamericana de Evaluación Educativa, 13(2), 67-83. Retrieved from https://dx.doi.org/10.15366/riee2020.13 .2.004 10.15366/riee2020.13.2.004

Bellucci, A., y Pennacchio, L. (2016). University knowledge and firm innovation: evidence from European countries. The Journal of Technology Transfer, 41(4), 730-752. Retrieved from https://dx.doi.org/10.1007/ s10961-015-9408-9 10.1007/s10961-015-9408-9

Calvo, R., y Rodríguez, J. A. (2014). La auditoría sociolaboral: una herramienta por descubrir (y utilizar). Lan harremanak: Revista de relaciones laborales, 30, 71-93.

Calvo, R., Rodríguez, J. A., Lorente, R., Benedito, M. A., Cantarero, S., y Cano, F. J. (2017). INVESLAB: Innovación e investigación en Ciencias Laborales desde y para las Ciencias Laborales (E. F. C. y R. Calvo, Ed.).

Calvo-Palomares, R., Payá-Castiblanque, R., y Sigalat-Signes, E. (2019). Uso de herramientas profesionales en la docencia universitaria. Una experiencia en ciencias laborales. Revista Perspectiva Empresarial, 6(2), 59-73. Retrieved from https://dx.doi.org/10.16967/23898186.601 10.16967/23898186.601

Eirín, R., García, H. M., y Montero, L. (2009). Desarrollo profesional y profesionalización docente. Perspectivas y problemas. Profesorado. Revista de Currículum y Formación de Profesorado, 13(2), 1-13.

Gento-Palacios, S., y Vivas-García, M. (2003). El SEUE: un instrumento para conocer la satisfacción de los estudiantes universitarios con su educación. Acción Pedagógica, 12(2), 16-27.

González-López, I. (2003). Determinación de los elementos que condicionan la calidad de la universidad: Aplicación práctica de un análisis factorial. RELIEVE, 9(1), 83-96. https://doi.org/10.7203/relieve.9.1.4351

Grañeras, M., y Parras, A. (2008). Orientación educativa: Fundamentos teóricos, modelos y nuevas perspectivas. Madrid: Ministerio de Educación, Política Social y Deporte.

Grey, C. (1997). Management as a technical practice: Professionalization or responsibilization. Systems Practice, 10, 703-725.

Hewitt-Dundas, N., Gkypali, A., y Roper, S. (2019). Does learning from prior collaboration help firms to overcome the 'two-worlds' paradox in university-business collaboration? Research Policy, 48(5), 1310-1322. Retrieved from https://dx.doi.org/10.1016/j.respol.2019.01.016 10.1016/j.respol.2019.01.016

INVESLAB. (n.d.). INVESLAB. Grupo de Innovación e Investigación en CC. Laborales de la Universidad de Valencia. https://inveslab.blogs.uv.es/. Retrieved from https://inveslab.blogs.uv.es/

Jiménez-González, A., Terriquez-Carrillo, B., y Zepeda, F. J. R. (2011). Evaluación de la satisfacción académica de los estudiantes de la Universidad Autónoma de Nayarit. Revista Fuente, 2(6), 46-56.

López-Martínez, A., y Mena-Lorenzo, J. (2016). La orientación profesional en la especialidad Gestión del Capital Humano. Mendive. Revista de Educación, 14(1), 56-64.

Mahecha, J. (2012). Profesionalización y profesionalismo en la docencia escolar. Retrieved from https:// blogs.iadb.org/educacion/es/profesionalizacion-y-profesionalismo-en-la-docencia-escolar/

Mejías, A., y Martínez, D. (2009). Desarrollo de un instrumento para medir la Satisfacción Estudiantil en Educación Superior. Docencia Universitaria, 10(2), 29-47.

Ortiz-Ocaña, A. L. (2003). El aprendizaje profesionalizado. Una opción para el desarrollo de competencias laborales en el proceso pedagógico profesional. www.monografías.com. Retrieved from https://www .monografias.com/trabajos26/competencias-laborales/competencias-laborales.shtml

Ortiz-Ocaña, A. L. (2011). Hacia una nueva clasificación de los modelos pedagógicos: El pensamiento configuracional como paradigma científico y educativo del siglo XX. Praxis, 7(1), 121-137.

Pedró, F. (2020). ¿Ofrecen las universidades una formación inicial docente suficientemente profesionalizadora? Revista Educación Superior y Sociedad, 32(1), 60-88.

Pérez-Juste, R. (2000). La calidad de la educación. In R. Pérez, F. López, M. Peralta, y P. Municio (Eds.), Hacia una educación de calidad. Gestión, instrumentos y evaluación (p. 13-44). Madrid: Narcea.

Prat, R., y Doval, E. (2005). Construcción y análisis estadístico de escalas. In J. Lèvy y J. Valera (Eds.), Análisis Multivariante para las Ciencias Sociales (p. 43-89). Madrid: Pearson Prentice Hall.

Sánchez, A. V., y Leicea, O. V. (2007). Competence-based learning and the development of the social dimension in universities. Educar, 40, 15-15. 10.5565/rev/educar. 149

Saraiva, M. (2008). La calidad y los "clientes" de la enseñanza superior Portuguesa. Horizontes Educacionales, 13(2), 41-54.

Secadas, F. (1962). Sentido y problemas del aprendizaje profesional. Revista de Educación-Estudios, 147, 6-11.

Torío-López, S., Peña-Calvo, J. V., y Fernández-García, C. M. (2010). Evaluación de una experiencia de 
innovación docente para el aprendizaje significativo en pedagogía: un marco para la reflexión a partir de la percepción de los estudiantes. ESE. Estudios sobre educación, 18, 229-254.

Trostinskaia, I. R., Safonova, A. S., y Pokrovskaia, N. N. (2017). Professionalization of education within the digital ecnomy and communicative competencies. IEEE VI Forum Strategic Partnership of Universities and Enterprises of Hi-Tech Branches, 29-32. 